BULL. AUSTRAL. MATH. SOC.

VOL. 17 (1977), 479-480.

\title{
An embedding theorem for ordered groups: Addendum
}

\section{Colin D. Fox}

A simple argument yields the following generalization of Theorem 6 of [1] (whose notation is retained without further explanation).

THEOREM. Let $G$ be an o-group and $\underline{\underline{V}}$ a variety of groups. Then $G \in \underline{\underline{\mathrm{V}}}$ implies $G^{\#} \in \underline{\underline{\mathrm{V}}}$.

Proof. Suppose $W\left(x_{1}, \ldots, x_{n}\right)$ is a law of $\underline{\underline{V}}$ and take

$$
\begin{aligned}
& \left(g_{1}, a_{1}\right), \ldots,\left(g_{n}, a_{n}\right) \text { in } G^{\#} \text {. So } \\
& w\left(\left(g_{1}, a_{1}\right), \ldots,\left(g_{n}, a_{n}\right)\right)=\left(w\left(g_{1}, \ldots, g_{n}\right), W^{\prime}\left(b_{1} \phi_{h_{1}}^{\#}, \ldots, b_{r} \phi_{h_{r}}^{\#}\right)\right) \\
& =\left\{1, W^{\prime}\left(b_{1} \phi_{h_{1}}^{\#}, \ldots, b_{r} \phi_{h_{r}}^{\#}\right)\right\}
\end{aligned}
$$

for some word $W^{\prime}\left(x_{1}, \ldots, x_{n}\right\}$, and where each $b_{i} \in\left\{a_{1}, \ldots, a_{n}\right\}$ and $h_{i} \in G$.

$$
\text { Setting } \begin{aligned}
m & =m\left(a_{i}\right), i=1,2, \ldots, n, \text { we have, in } G, \\
1 & =W\left(g_{1} a_{1}^{m}, \ldots, g_{n} a_{n}^{m}\right)=W^{\prime}\left(b_{1}^{m} \phi_{h_{1}}, \ldots, b_{r}^{m} \phi_{h_{r}}\right) .
\end{aligned}
$$

Hence in $G^{\#}$,

$$
l=\left(1, W^{\prime}\left(b_{1}^{m} \phi_{h_{1}}, \ldots, b_{r}^{m} \phi_{h_{p}}\right)\right)=\left(1, W^{\prime}\left(b_{1} \phi_{h_{1}}^{\#}, \ldots, b_{p} \phi_{h_{p}}^{\#}\right)\right)^{m} .
$$

Since $G^{\#}$ is torsion-free, $W\left(\left(g_{1}, a_{1}\right), \ldots,\left(g_{n}, a_{n}\right)\right)=1$ in $G^{\#}$.

Received 27 September 1977. 


\section{Reference}

[1] Colin D. Fox, "An embedding theorem for ordered groups", BulZ. Austral. Math. Soc. 12 (1975), 321-335.

Department of Pure Mathematics,

La Trobe University,

Bundoora,

victoria. 